\title{
The Role of Nav1.8 in Cardiac Electrophysiology-a Matter of the Heart or the Nerve?
}

\author{
Katja E. Odening ${ }^{1}$ \\ Published online: 18 January 2020 \\ (C) Springer Science+Business Media, LLC, part of Springer Nature 2020
}

$S C N 5 A /$ Nav1.5 is the canonical voltage-gated cardiac sodium channel conducting depolarizing $\mathrm{I}_{\mathrm{Na}}$ current in the heart. Moreover, SCN5A/Nav1.5 channels harboring gain-offunction mutations with impaired inactivation or pathologically over expressed/over activated SCN5A/Nav1.5 channels conduct pathological late $\mathrm{I}_{\mathrm{Na}, \mathrm{L}}$, which impairs repolarization in the heart - relevant in a variety of different cardiac arrhythmogenic diseases such as long QT syndrome, heart failure, and cardiac hypertrophy (1-3). SCN10A/Nav1.8, in contrast, is an important voltage-gated sodium channel conducting depolarizing $\mathrm{I}_{\mathrm{Na}}$ current in the nervous system - particularly in dorsal root ganglia, nociceptive nerves, and cardiac nerves/ ganglionated plexi (4-6).

This classical distinction between cardiac and neuronal sodium channel isoforms, however, has been challenged in recent years: the potential role for the neuronal $S C N 10 \mathrm{~A} / \mathrm{Nav} 1.8$ in modulating cardiac electrophysiology in health and disease and its potential impact on arrhythmia susceptibility is an ongoing matter of debate. Genome-wide association studies have associated variants in SCN1OA/Nav1.8 with alterations of cardiac conduction $(7,8)$ and with atrial fibrillation $(9,10)$. SCN10A/Nav1.8 mutations have been identified in Brugada syndrome $(11,12)$. Moreover, recently, it has been demonstrated that $S C N 10 A / \mathrm{Nav} 1.8$ is upregulated in heart failure (13) and cardiac hypertrophy (14), thus contributing to electrical remodeling in diseased hearts. All these studies suggest that $S C N 10 A /$ Nav1.8 may play a role in arrhythmogenic cardiac diseases.

But is the channel expressed in the heart or rather in cardiac neurons? And is it thus exerting direct effects on cardiac

Katja E. Odening

katja.odening@uniklinik-freiburg.de

1 Department of Cardiology and Angiology I and Institute of Experimental Cardiovascular Medicine, Heart Center University of Freiburg, Medical Faculty, University of Freiburg, Hugstetter Str. 55, 79106 Freiburg, Germany electrophysiology or are its effects mediated via the nervous system?

In their study in this issue, Casini, Remme, and colleagues aimed at elucidating whether SCN10A/Nav1.8 is expressed and functionally active in the healthy heart (15). In a thorough approach, they investigated the functional relevance of SCN10A/Nav1.8 in different types of cardiomyocytes (CMs) (e.g., atrial and ventricular $\mathrm{CMs}$ ), and in different species (e.g., rabbit left ventricular $\mathrm{CMs}$, human left atrial $\mathrm{CMs}$, and human-induced pluripotent stem cells derived cardiomyocytes (hiPSC-CMs)) - focusing on the contribution of Nav1.8 to both peak and late sodium current under normal, physiological conditions. In cardiomyocytes from all different species investigated, resting membrane potentials, action potential (AP) amplitudes, and AP upstroke velocities were all unaffected by the Nav1.8-blocker A-803467 and Nav1.8-based late sodium current was undetectable. Similarly, Nav1.8-blockade had no effect on APD in human atrial cells - while a slight shortening of APD was observed in rabbit CMs and hiPSC$\mathrm{CMs}$; likely due to some time-dependent run-down in the latter cells. All these patch clamp findings indicate no (relevant) functional role of Nav1.8 in the healthy myocardium. To complement their functional assessment, the authors investigated cardiac mRNA expression levels of SCN10A, which were found to be low to absent in human atrial tissue, rabbit ventricular tissue, and hiPSC-CMs, further supporting the notion that SCN1OA/Nav1.8 might not be important for the physiological electrical function in the healthy heart.

Important to note, to verify their findings and conclusions, the authors have performed several additional studies investigating the validity of the employed methods (15). To check whether run-down may underlie (in part) the apparent minor effects of the Nav1.8-blocker on APD in some of their experiments and the fact that these effects were not reversible upon washout, the authors have included vehicle time-matched control measurements. These controls clearly demonstrate shortening of APD over time - similar as observed in the presence of 
the Nav1.8-blocker, supporting the notion that the observed apparent small shortening of APD induced by A-803467 in rabbit CMs is likely a time-dependent effect independent of the blocker. These experiments clearly highlight the importance of running time-matched controls. Moreover, to verify that Nav1.8-blocker A-803467 is indeed working on Nav1.8-mediated sodium current, the authors have additionally recorded action potentials (APs) from murine intracardiac neurons in the presence and absence of Nav1.8-blocker A-803467, demonstrating its general functionality on neuronal $\mathrm{I}_{\mathrm{Na}}$ : in the presence of A-803467, AP upstroke velocity was reduced, an effect that was reversible upon washout $(5,15)$.

Transcription of SCN10A was first investigated in human iPSC-CMs and human left atrial tissue and compared with SCN5A mRNA levels in the same samples. The authors have then elegantly complemented these data with SCN5A and SCN10A mRNA expression data in other cardiac tissues (rabbit left ventricle, hiPSC-CMs, and human right and left atria), which they have extrapolated from analyses of online RNA sequencing raw datasets previously published, providing a nice example of how published raw data can be reused for different research questions.

Support for the observation by Casini and colleagues of a virtually absent expression of $S C N 10 A$ in healthy rabbit, mouse, and human cardiomyocytes (15) has recently been published by Gando et al. (16). In this study, no Nav1.8 protein or mRNA expression was detected in the specialized cardiac conduction system of mice, and rat and human ventricles - even in subjects with sudden unexplained death and pathogenic SCN1OA mutations, suggesting that the effect of $S C N 10 A / N a v 1.8$ on cardiac electrical function is likely to be extra-cardiac in origin.

One prime candidate is the nervous system; and indeed, several studies have investigated and verified an effect of Nav1.8-blockade in cardiac ganglionated plexi/intracardiac neurons on cardiac electrophysiology, cardiac conduction, and $\mathrm{AF}$ inducibility $(5,6,17)$. Another mechanism by which SCN10A may impact on cardiac electrophysiology - f. ex. in Brugada syndrome - is via a genetic interaction with SCN5A (via enhancer-promotor interactions between SCN1OA and SCN5A loci) (18).

In contrast to these findings in healthy, structurally normal hearts and cardiomyocytes, in disease states - such as heart failure and cardiac hypertrophy - a significant and relevant upregulation of $S C N 10 \mathrm{~A} / \mathrm{Nav} 1.8 \mathrm{mRNA}$ and protein expression has been observed both in cardiac tissue and in cardiomyocytes and its functional relevance has been verified by blocking experiments $(13,14)$, suggesting that in the diseased, remodeled heart, the neural form Nav1.8 may indeed directly affect the cardiomyocyte's electrical function.

The role of SCN10A/Nav1.8 in cardiac electrical function is thus not a matter of the heart or the nerve but rather of bothdepending on the heart's state.

\section{References}

1. Wang Q, Shen J, Splawski, et al. SCN5A mutations associated with an inherited cardiac arrhythmia, long QT syndrome. Cell 1995 Mar 10;80(5):805-811. https://doi.org/10.1016/0092-8674(95)90359-3

2. Horvath B, Bers DM. The late sodium current in heart failure: pathophysiology and clinical relevance. ESC Heart Fail. 2014 Sep;1(1):26-40. https://doi.org/10.1002/ehf2.12003.

3. Toischer K, Hartmann N, Wagner S, Fischer TH, Herting J, Danner $\mathrm{BC}$, et al. Role of late sodium current as a potential arrhythmogenic mechanism in the progression of pressure-induced heart disease. $\mathrm{J}$ Mol Cell Cardiol. 2013 Aug;61:111-22. https://doi.org/10.1016/j. yjmcc.2013.03.021.

4. Renganathan M, Cummins TR, Waxman SG. Contribution of $\mathrm{Na}(\mathrm{v}) 1.8$ sodium channels to action potential electrogenesis in DRG neurons. J Neurophysiol. 2001 Aug;86(2):629-40. https:// doi.org/10.1152/jn.2001.86.2.629

5. Verkerk AO, Remme CA, Schumacher CA, Scicluna BP, Wolswinkel R, de Jonge B, et al. Functional Nav1.8 channels in intracardiac neurons: the link between SCN10A and cardiac electrophysiology. Circ Res. 2012 Jul 20;111(3):333-43. https://doi. org/10.1161/CIRCRESAHA.

6. Chen X, Yu L, Shi S, et al. Neuronal Nav1.8 channels as a novel therapeutic target of acute atrial fibrillation prevention. J Am Heart Assoc. 2016 Nov 2;5(11). pii: e004050.

7. Delaney JT, Muhammad R, Shi Y, Schildcrout JS, Blair M, Short L, et al. Common SCN10A variants modulate PR interval and heart rate response during atrial fibrillation. Europace. 2014 Apr;16(4): 485-90. https://doi.org/10.1093/europace/eut278.

8. Macri V, Bridy JA, Arking DE, et al. Common coding variants in SCN10A are associated with the Nav1.8 late current and cardiac conduction. Circ Genom Precis Med. 2018 May;11(5):e001663. doi: https://doi.org/10.1161/CIRCGEN.116.001663.

9. Jabbari J, Olesen MS, Yuan L, Nielsen JB, Liang B, Macri V, et al. Common and rare variants in $\mathrm{SCN} 10 \mathrm{~A}$ modulate the risk of atrial fibrillation. Circ Cardiovasc Genet. 2015 Feb;8(1):64-73. https:// doi.org/10.1161/HCG.0000000000000022.

10. Savio-Galimberti E, Weeke P, Muhammad R, Blair M, Ansari S, Short L, et al. SCN10A/Nav1.8 modulation of peak and late sodium currents in patients with early onset atrial fibrillation. Cardiovasc Res. 2014 Nov 1;104(2):355-63. https://doi.org/10.1093/cvr/ cvu170.

11. Bezzina CR, Barc J, Mizusawa Y, Remme CA, Gourraud JB, Simonet F, et al. Common variants at SCN5A-SCN10A and HEY2 are associated with Brugada syndrome, a rare disease with high risk of sudden cardiac death. Nat Genet. 2013 Sep;45(9): 1044-9. https://doi.org/10.1038/ng.2712.

12. Hu D, Barajas-Martínez H, Pfeiffer R, Dezi F, Pfeiffer J, Buch T, et al. Mutations in SCN10A are responsible for a large fraction of cases of Brugada syndrome. J Am Coll Cardiol. 2014 Jul 8;64(1): 66-79. https://doi.org/10.1016/j.jacc.2014.04.032

13. Dybkova N, Ahmad S, Pabel S, Tirilomis P, Hartmann N, Fischer TH, et al. Differential regulation of sodium channels as a novel proarrhythmic mechanism in the human failing heart. Cardiovasc Res. 2018 Nov 1;114(13):1728-37. https://doi.org/10.1093/cvr/ cvy 152 .

14. Ahmad S, Tirilomis P, Pabel S, Dybkova N, Hartmann N, Molina $\mathrm{CE}$, et al. The functional consequences of sodium channel $\mathrm{Na}_{\mathrm{V}} 1.8$ in human left ventricular hypertrophy. ESC Heart Fail. 2019 Feb;6(1):154-63. https://doi.org/10.1002/ehf2.12378.

15. Casini S, Marchal GA, Kawasaki M, et al. Absence of functional Nav1.8 channels in non-1 diseased atrial and ventricular cardiomyocytes. Cardiovasc Drugs Ther. 2020; 33(6). https://doi. org/10.1007/s10557-019-06931-8. 
16. Gando I, Williams N, Fishman GI, Sampson BA, Coetzee WA. Functional characterization of SCN10A variants in several cases of sudden unexplained death. Forensic Sci Int. 2019 Aug;301: 289-98. https://doi.org/10.1016/j.forsciint.2019.05.042.

17. Qi B, Wei Y, Chen S, Zhou G, Li H, Xu J, et al. Nav1.8 channels in ganglionated plexi modulate atrial fibrillation inducibility. Cardiovasc Res. 2014 Jun 1;102(3):480-6. https://doi.org/10. 1093/cvr/cvu005
18. van den Boogaard M, Smemo S, Burnicka-Turek O, Arnolds DE, van de Werken $\mathrm{H}$, Klous $\mathrm{P}$, et al. A common genetic variant within SCN10A modulates cardiac SCN5A expression. J Clin Invest. 2014 Apr;124(4):1844-52. https://doi.org/10.1172/JCI73140

Publisher's Note Springer Nature remains neutral with regard to jurisdictional claims in published maps and institutional affiliations. 